\title{
Nematicon-driven injection of amplified spontaneous emission into an optical fiber
}

\author{
Serena Bolis ${ }^{1,2, *}$, Tersilla Virgili ${ }^{3}$, Sai Kiran Rajendran ${ }^{3, \dagger}$, Jeroen BeEckman ${ }^{2}$, ANd \\ PASCAL KOCKAERT ${ }^{1}$ \\ ${ }^{1}$ Université libre de Bruxelles (U.L.B.), OPERA-photonics group, CP 194/5, 50 Avenue F.D. Roosevelt, 1050 Bruxelles, Belgium \\ ${ }^{2}$ Ghent University, ELIS Department, Technologiepark-Zwijnaarde 15, 9052 Gent, Belgium \\ ${ }^{3}$ IFN-CNR, Dipartimento di Fisica, Politecnico di Milano, Piazza Leonardo da Vinci 32, 20132 Milano, Italy \\ ${ }^{\dagger}$ current address: School of Physics and Astronomy, University of St. Andrews, St. Andrews, Fife, KY16 9SS (UK) \\ *Corresponding author: Serena.Bolis@ulb.ac.be

We investigate experimentally the interaction between amplified spontaneous emission (ASE) and a soliton, which are both generated in a dye-doped nematic liquid crystal (LC) cell. A light beam is injected through an optical fiber slid into the cell to form a soliton beam. ASE is then automatically collected by this self-induced waveguide and efficiently coupled into the same optical fiber, in the backward direction. We demonstrate that the presence of the soliton improves the ASE collection by one order of magnitude. We also show that the ASE is highly polarized in the plane of the LC cell, and that the ASE spectrum depends on the pump stripe orientation with respect to the LC director. The origin of the spectral anisotropy of the gain curves is determined with the help of femtosecond pump-probe spectroscopy. (๑ 2016 Optical Society of America

OCIS codes: (160.3710) Liquid crystals; (190.6135) Spatial solitons; (300.6530) Spectroscopy, ultrafast.

http://dx.doi.org/10.1364/OL.41.002245

Amplified spontaneous emission (ASE) arises in gain media that are excited with a stripe-shaped beam, like in fiber amplifiers or bulk amplifying materials. Photons that are emitted spontaneously along the long axis of the excited area are amplified more than those emitted in other directions. The ASE is therefore directional and narrow-band, since during the propagation the wavelength with the highest optical gain emerges from the photo-luminescence broadband spectrum [1-4]. The absence of feedback simplifies the architecture of the device with respect to lasers and is also responsible for a low spatial coherence. ASE is therefore a better candidate for illumination purposes than broadband and narrow-band lasers, which are impaired by speckle $[5,6]$.

ASE has been widely studied in solid state films [7-11], microfluidic devices $[12,13]$ and only recently in dye-doped nematic liquid crystals (LCs) [14, 15]. Nematic LCs are character- ized by a mean orientation of the molecules, called director. Rodlike LCs exhibit anisotropic permittivity, with a different value parallel $\left(\varepsilon_{\|}\right)$and orthogonal $\left(\varepsilon_{\perp}\right)$ to the long axis of the molecules. When the anisotropy is positive $\left(\varepsilon_{\|}>\varepsilon_{\perp}\right)$, LC molecules are reoriented parallel to an applied external electric field. In particular, a light beam can reorient the LC director locally, which induces a local increase of the refractive index. The beam propagates without diffraction as it is confined by the self-induced waveguide. This soliton-like beam is called nematicon, as it occurs in nematic LCs [16-18]. Once the nematicon is formed, it can be used as waveguide to transmit other beams [16]. In our case the nematicon is generated in a dye-doped LC cell. Elongated molecules, like dyes or polymers, which are dispersed in nematic liquid crystal host show the tendency to align themselves along the director $[19,20]$. The ordering imprinted by the LC also increases the pumping efficiency and the polarization ratio of the emission. The main drawback of LC as host for photoluminescent materials is their need to be integrated into glass cells, in order to have a macroscopic order of the LC. The edges of the cell are either sealed with glue or left open, in both cases a depolarization and a de-focusing of the ASE beam take place.

In this letter we propose a new way to efficiently collect and extract the ASE from a LC cell through the combined action of a nematicon and an optical fiber slid into the cell. The nematicon waveguide induced at the fiber end collects the ASE and guides it back into the fiber and then outside of the device where it can be used for applications, like incoherent lighting. The ASE guided by the nematicon does not spread along the propagation direction, in this way it is better injected into the fiber with respect to a diffracted beam. We demonstrate that the presence of the soliton increases the intensity of the ASE collected by the fiber by one order of magnitude. Finally, by means of polarized ultra-fast spectroscopy, we show that the ASE wavelength tunability that we obtain as a function of the soliton power depends mainly on the waveguiding properties of the nematicon rather than on the gain anisotropy of the dye molecules.

We aim to generate ASE and collect it with a soliton beam. To this end, we use the setup depicted in Fig. 1, where green light is used to pump the gain medium dispersed in the LC cell. The nematicon is generated in the infrared from the output mode 


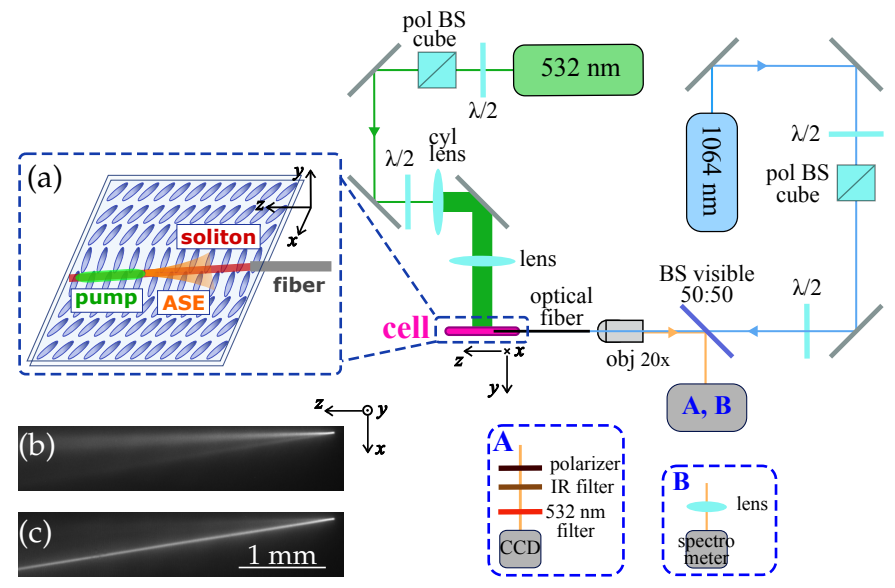

Fig. 1. Scheme of the nematicon injection and the ASE generation in the dye-doped nematic LC cell. (a) Scheme of the cell with the fiber slid inside it and the stripe-shaped pump coming orthogonal to the cell surface. The fiber, inserted from the right is horizontal and at $45^{\circ}$ to the rubbing direction. (b)(c) Propagation of the IR beam of $1.7 \mathrm{~mW}$ (without the pump beam) for a polarization parallel to the $y(b)$ or to the $x$ axis (c).

of an optical fiber inserted into the LC cell. Light collected in the backward direction is reflected on a beam-splitter, passes through spectral filters, and is finally sent to either a camera or a spectrometer for characterization. A particular challenge in this setup is to insert an optical fiber into a thin LC cell.

The LC cell is made of two Indium-Tin-Oxide (ITO) coated glass plates that are spin-coated with nylon and then rubbed in order to induce a preferential orientation of the LC director on these two surfaces. The distance between the two glass plates is fixed by spherical spacers $(75 \mu \mathrm{m})$ mixed with UV-curable glue. Once assembled, the cell is infiltrated by capillarity with a solution of 1 wt.\% of pyrromethene 597 dye (PM597, SigmaAldrich) in E7 LC (Merck). A non-standard optical fiber, with a cladding diameter of $64.4 \mu \mathrm{m}$, a core diameter of $2.9 \mu \mathrm{m}$, and a cut-off wavelength of $550 \mathrm{~nm}$, is slid inside the cell along the $z$ direction (Fig. 1a).

The two optical sources used to create the nematicon and to excite the dye are, respectively, a continuous wave Nd:YAG laser (1064 nm) and a Q-switched frequency doubled Nd:YAG laser (532 nm, 400-ps pulses at 10 or $100 \mathrm{~Hz}$ ). On both arms, the first half-wave plate and the polarizing beam splitter allow to control the beam power while the second half-wave plate defines the input polarization angle with respect to the LC director. We set the polarization of the green beam (pump) to maximize the fluorescence and we align the polarization of the IR beam (soliton) along $x$.

The IR beam is injected into the optical fiber through a microscope objective $(\mathrm{f}=8.0 \mathrm{~mm}, \mathrm{NA}=0.5)$. Indeed, since the rubbing direction is at $45^{\circ}$ with respect to the fiber injection, the torque of the optical electric field on the LC director is minimum if the input beam is polarized along $y$. In this case the director does not reorient and the beam diffracts (Fig. 1b). If the input beam is polarized along $x$, the optical torque is maximized and the power needed to create a nematicon is minimized (Fig. 1c) [17, 21, 22]. The nematicon propagates with an optical walk-off of around $8^{\circ}$ with respect to the $z$ axis due to the optical anisotropy of the LC [23]. The stripe-shaped green beam is therefore also tilted to maximize the overlap with the nematicon (Fig. 1a). This beam is focused with a spherical achromatic and a cylindrical lens in order to obtain an elliptical spot of around $20 \mu \mathrm{m} \times 7 \mathrm{~mm}$ on the cell. The pump stripe is aligned to form a gap of around $2 \mathrm{~mm}$ between the pumping area and the end of the fiber. The ASE threshold is as low as $0.4 \mu \mathrm{J} /$ pulse, and all the measurements presented here are taken well above.

To characterize the ASE collected into the fiber, the beam is first collimated with the same microscope objective as used to inject the IR into the fiber, then reflected on a beam splitter for the visible light. This light is then characterized spatially, with the ensemble $532 \mathrm{~nm}$ notch/IR filters and a monochromatic CCD camera (JAI AM-800 GE, case A in Fig. 1), and spectrally, using a spectrometer (Ocean Optics USB2000+, case B in Fig. 1).

The far field intensity profiles of the ASE light collected by the nematicon and coming out of the fiber are shown in Fig. 2. In these measurements we separate the emission polarized along $x$ (parallel to the substrate surface, Fig. 1a) from that one polarized along $y$ (orthogonal to the substrate surface). Due to its high directionality, a small amount of the ASE is coupled into the fiber even if the soliton is not present (Fig. 2a-b). The power ratio between the two linear polarization components is $\sim 5.1$. A similar ratio $(\sim 4.4)$ is obtained if the end of the pump stripe is placed close to the fiber and the ASE does not travel inside the unexcited LC. The ASE is therefore mainly polarized in the plane of the substrate, as also reported for solid state films [24].

When an IR beam of $2.0 \mathrm{~mW}$ is injected into the cell through the fiber, it creates a nematicon that captures the ASE light and efficiently couples it into the fiber. The intensity profiles of the collected ASE are modified as reported in Fig. $2 \mathrm{c}-\mathrm{d}$. The
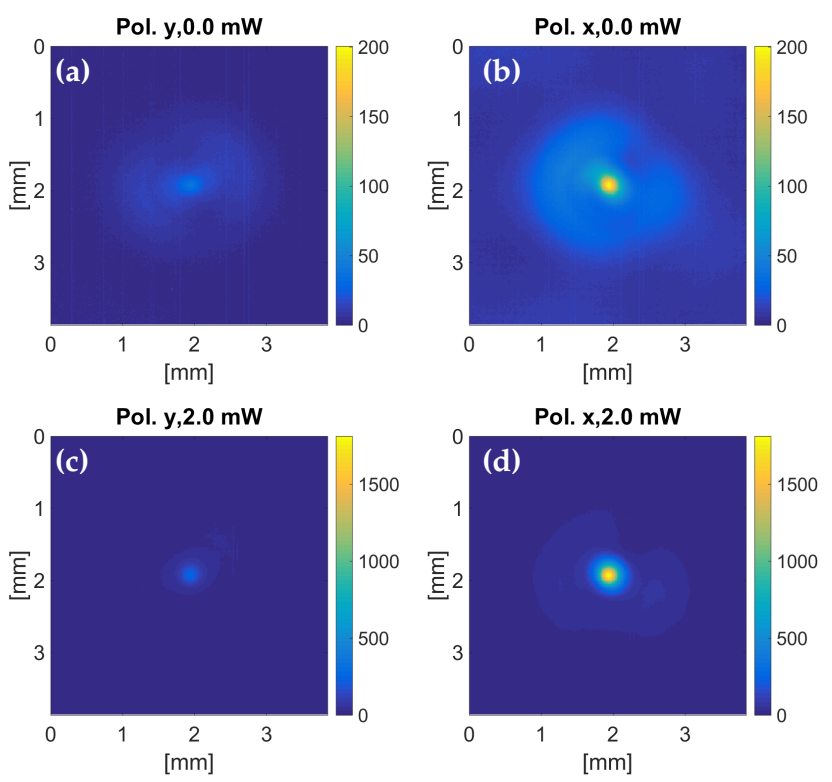

Fig. 2. ASE profile coming out of the fiber for the case (a)(b) without nematicon $(0 \mathrm{~mW})$ and $(\mathrm{c})-(\mathrm{d})$ with a nematicon $(2.0 \mathrm{~mW})$. The two polarizations, orthogonal (a)-(c) or parallel (b)-(d) to the substrate surface, are shown. The images are taken at $35 \mathrm{~cm}$ from the microscope objective and the pump delivers pulses of $2.0 \mu \mathrm{J} /$ pulse at $10 \mathrm{~Hz}$; each profile is an average of 100 images. The presence of the nematicon induces an increase of both the polarization ratio and the intensity of the collected ASE. 

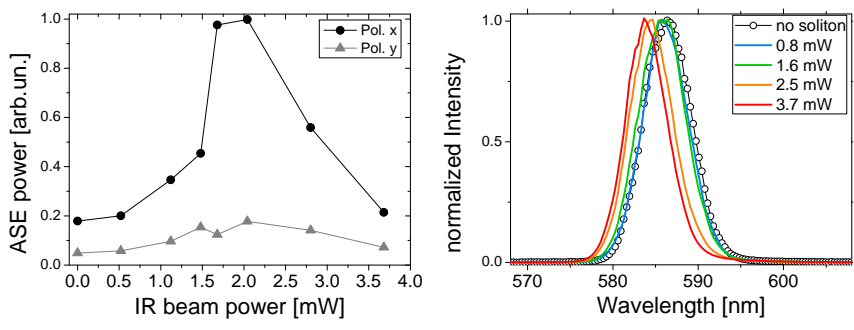

(a)

(b)

Fig. 3. (a)ASE collected power as a function of the IR beam power. The ASE power is obtained integrating the intensity profiles like those reported in Fig. 2. The pump delivers pulses of $2.0 \mu \mathrm{J} /$ pulse at $10 \mathrm{~Hz}$. (b) Normalized spectra collected at the output of the fiber at different IR beam powers; each spectrum is an average of 200 scans. The pump delivers pulses of $6.6 \mu \mathrm{J} /$ pulse at $100 \mathrm{~Hz}$.

waveguide induced by the soliton increases the collected light polarized along $x$ by almost one order of magnitude $(\sim 9.0)$ with respect to the case without nematicon. This factor is almost double that obtained by Henninot and co-workers with a thermal soliton in LC collecting non-directional fluorescence [25]. The power ratio between the two polarizations is also significantly increased $(\sim 7.0)$, due to the fact that the waveguide profile is created only for the light polarized along $x$ [16].

The power of the collected ASE for different IR beam powers is obtained by integrating the intensity profile at the end of the fiber and it is reported in Fig. 3a. At powers below 0.7 $\mathrm{mW}$, the nematicon is not formed. The ASE beam is therefore not guided along a preferential direction resulting in a small amount of ASE coupled into the fiber. Above this threshold, the nematicon is created. The ASE is guided by this self-induced waveguide up to the fiber, allowing an efficient coupling and causing an important increase of the intensity of the collected ASE. As the refractive index contrast of the waveguide increases with the soliton power, due to a stronger LC reorientation, the guiding efficiency increases also. Therefore, the intensity of the collected ASE increases with the nematicon power and reaches a maximum at $2 \mathrm{~mW}$, above which oscillations start to play an important role. Finally, at high IR beam powers the intensity profile of the nematicon starts to oscillate in the plane $x z$. Indeed at higher powers of the IR beam the soliton starts to overfocus: the beam waist becomes of the same order of magnitude of the thermal fluctuations of the refractive index and the nematicon is deviated from its path $[18,26]$. The spatial oscillations induce a rapid decrease in the efficiency of the ASE collection and waveguiding. The optimum ASE collection is therefore obtained at the maximum power for which the nematicon is not oscillating.

Using the configuration reported in Fig. 1 (case B), we analyze the spectrum of the light collected by the nematicon and guided into the optical fiber (Fig. 3b). The normalized spectra are acquired for the same pump intensity $(2.0 \mu \mathrm{J} /$ pulse $)$ and at different nematicon intensities. Quite interestingly, we observe a blue-shift of the ASE peak ( $\sim 3 \mathrm{~nm})$ at high soliton powers, when the collected power is also decreasing due to the nematicon oscillations. To explain this blue-shift, we consider two phenomena. On the one hand, the reorientation of the dye molecules together with the LC can modify the ASE spectrum, due to the anisotropy of the optical gain. On the other hand, at high nematicon powers, the soliton beam starts to oscillates in space due to thermal fluctuations, inducing wavelength-dependent bend losses [27].

In order to identify the relative contribution of the gain anisotropy and of the waveguide losses to the observed spectral shift, we characterize the photophysical properties of the mixture by means of femtosecond pump-probe spectroscopy. In particular this technique allows to measure the spectral and the temporal evolution of the optical gain of a material. In our case, the classical pump-probe setup was improved in order to separate the stimulated emission parallel and orthogonal to the LC director and to study the optical anisotropy of the sample [28]. In the setup, pulses generated by a mode-locked Ti:sapphire oscillator are stretched, amplified and then compressed to provide pulses centered at $800 \mathrm{~nm}$, with a duration of $100 \mathrm{fs}$, and an energy above $1 \mathrm{~mJ}$ per pulse, at a repetition rate of $1 \mathrm{kHz}$. A first part of this beam is sent into an optical parametric amplifier to generate pump pulses centered at $550 \mathrm{~nm}$ with an energy of $10 \mathrm{~nJ} /$ pulse. The polarization of the pump beam is set parallel to the LC director and focused onto a circular spot of around $100 \mu \mathrm{m}$ diameter. A second part of the beam is focused onto a sapphire crystal to generate white light continuum (probe pulses). Both beams are focused at the same spot on the sample with a variable delay denoted by $\tau$. The polarization of the probe beam, defined by the orientation of the half-wave plate before the sapphire plate, is set either parallel or orthogonal to the LC director. A polarizer with optical axis parallel to the polarization of the incoming probe beam is placed after the cell in order to suppress the scattered light. The differential transmission of the probe through the excited sample is recorded with a spectrometer as a function of both the wavelength and the delay $\tau$

$$
\frac{\triangle T}{T}=\frac{T^{e x}-T^{\text {unex }}}{T^{\text {unex }}}(\lambda, \tau),
$$

where $T^{e x}$ and $T^{u n e x}$ are the transmissions of the probe through, respectively, the excited and unexcited sample. Positive signals are due either to ground state photo-bleaching (GSB), in the spectral region of the optical absorption, or to stimulated emission (SE) in the spectral region of the fluorescence. The absorption and emission of PM597 are centered respectively at $530 \mathrm{~nm}$ and $570 \mathrm{~nm}[29]$.

The samples for the pump-probe measurements are commercial cells (Instec), with a thickness of $6.8 \mu \mathrm{m}$, and a planar antiparallel alignment. They are filled with the same mixture as above. We use thinner samples than those used in the previous experiment of ASE generation in order to limit the depolarization of the beams during the propagation in such a scattering medium like LCs and also to minimize the absorption of the pump and the probe through the sample.

In Fig. 4 we report the pump-probe spectra of the mixture at 400 ps delay, with $10 \mathrm{~nJ} /$ pulse, for the two polarizations of the probe beam. The contribution polarized parallel to the rubbing is two times higher than the orthogonal one, indicating that the dye molecules are aligned along the LC director. Moreover the parallel contribution of both GSB (below $550 \mathrm{~nm}$ ) and SE (above $550 \mathrm{~nm}$ ) is shifted towards the longer wavelengths by around $10 \mathrm{~nm}$. In the same graph, we report also the ASE spectra for the cases where the axis of the pump stripe is set parallel or orthogonal to the rubbing direction. The scattered light is collected at the edge of the stripe from the large window of the cell ( $x z$ plane). Since the ASE emission is mainly polarized in the plane of the cell, and orthogonal to the Poynting vector, when the pumping stripe is parallel (resp. orthogonal) to the rubbing, the ASE is polarized mainly orthogonal (resp. parallel) to the director (Inset Fig. 4). Changing the emission polarization from 
orthogonal to parallel with respect to the director, the ASE peak shifts from $577 \mathrm{~nm}$ to $588 \mathrm{~nm}$. This red-shift is of the same order of magnitude as the shift observed in the pump-probe spectra. For this reason we believe that the large red-shift of the ASE is only to a minor part due to self-absorption anisotropy (higher for the light polarized parallel to the dye axis), while it is mostly due to the optical gain anisotropy of PM597 dissolved in LC.

The spectral behavior reported in Fig. $3 b$ is therefore not due to the anisotropy of the optical gain of the dye, since the spectral shift that we measure with pump-probe spectroscopy is in the opposite direction to the one observed with the presence of the soliton (Fig. 3b). Indeed, when the soliton is present, i.e. when the LC molecules are aligned more parallel to the electric field, we observe a blue-shift, instead of a red-shift as reported in Fig. 4. The observed blue-shift is therefore due to the change in the waveguiding properties of the soliton as a function of the power. The oscillations of the nematicon at high powers can be seen as a bending of the waveguide. Since the bend losses are higher for the longer wavelengths [27], the soliton oscillations act as a spectral filter, inducing a blue-shift in the collected ASE.

Finally, it is important to notice that both the nematicon waveguiding and the injection into the optical fiber act as filters for the non-directional and non-polarized light, eliminating the broad-band photo-luminescence spectrum present in Fig. 4 and leaving only the ASE peak (Fig. 3b).

We have demonstrated experimentally that the ASE collection from a LC device can be increased by one order of magnitude with the help of a nematicon that acts as a waveguide between the pump stripe where the ASE is generated and the fiber input face. The nematicon waveguiding property improves the spectral purity and the polarization degree of the collected light and it also introduces a small spectral shift, which does not come from the gain anisotropy and seems to result from bending losses appearing when the soliton starts to oscillate. Once

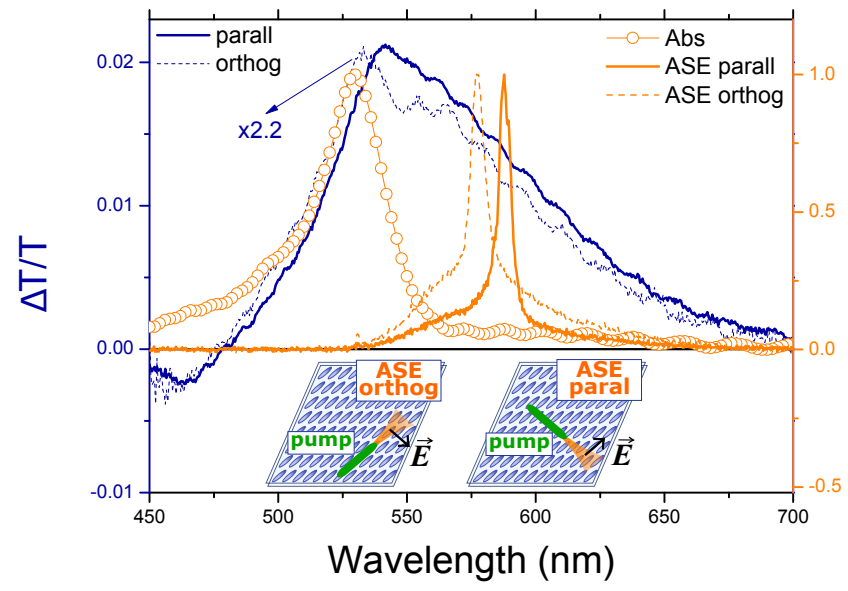

Fig. 4. Pump-probe spectra (blue) of the cell filled with a mixture of 1 wt.\% of PM597 in E7, taken at 400 ps delay for a pump of $10 \mathrm{~nJ} /$ pulse. The parallel spectrum (solid) is around two times higher than the orthogonal one (dashed). Normalized absorption spectrum (orange circles) for a polarization orthogonal to the rubbing (the parallel contribution saturates). The normalized ASE spectra (orange lines) are taken for a pump intensity of $7.6 \mu \mathrm{J} /$ pulse $(100 \mathrm{~Hz})$ for the cases where the electric field of the emission is parallel (solid) or orthogonal (dashed) to the rubbing direction. Inset: scheme of the ASE measurement in the two configurations. the polarized ASE light is collected into the fiber, it can easily be used for applications that require integrated and compact incoherent light sources, like lab-on-chip imaging.

Funding. Fonds pour la Formation à la Recherche dans l'Industrie et dans l'Agriculture (FRIA); Belgian Science Policy Office (BELSPO) (IAP7-35); Accordo Quadro Regione Lombardia-CNR.

\section{REFERENCES}

1. O. Svelto, S. Taccheo, and C. Svelto, Opt. Commun. 149, 277 (1998).

2. S. Stagira, M. Nisoli, G. Cerullo, M. Zavelani-Rossi, S. D. Silvestri, G. Lanzani, W. Graupner, and G. Leising, Chem. Phys. Lett. 289, 205 (1998).

3. J. Ribierre, G. Tsiminis, S. Richardson, G. Turnbull, I. Samuel, H. Barcena, and P. Burn, Appl. Phys. Lett. 91, 081108 (2007).

4. G. Tsiminis, N. Montgomery, A. Kanibolotsky, A. Ruseckas, I. Perepichka, P. Skabara, G. Turnbull, and I. Samuel, Semicond. Sci. Technol. 27, 094005 (2012).

5. B. Redding, M. Choma, and H. Cao, Nat. Photonics 6, 355 (2012).

6. B. Redding, P. Ahmadi, V. Mokan, M. Seifert, M. A. Choma, and H. Cao, Opt. Lett. 40, 4607 (2015).

7. M. D. McGehee, R. Gupta, S. Veenstra, E. K. Miller, M. A. Díaz-García, and A. Heeger, Phys. Rev. B 58, 7035 (1998).

8. G. Heliotis, D. Bradley, G. Turnbull, and I. Samuel, Appl. Phys. Lett. 81, 415 (2002).

9. F. Lahoz, C. J. Oton, N. Capuj, M. Ferrer-González, S. Cheylan, and D. Navarro-Urrios, Opt. Express 17, 16766 (2009).

10. E. Calzado, M. Ramírez, P. Boj, and M. Díaz-García, Appl. Opt. 51, 3287 (2012).

11. S. Bolis, M. Pasini, and T. Virgili, Chem. Commun. 49, 11761 (2013).

12. K. Vishnubhatla, J. Clark, G. Lanzani, R. Ramponi, R. Osellame, and T. Virgili, Appl. Phys. Lett. 94, 041123 (2009).

13. R. M. Gerosa, A. Sudirman, L. d. S. Menezes, W. Margulis, and C. J. de Matos, Optica 2, 186 (2015).

14. L. Blinov, G. Cipparrone, V. Lazarev, P. Pagliusi, and T. Rugiero, Opt. Express 16, 6625 (2008).

15. R. Fan, Y. Jiang, Y. Xia, and D. Chen, J. Russ. Laser Res. 32, 139 (2011).

16. M. Peccianti, A. D. Rossi, G. Assanto, A. D. Luca, C. Umeton, and I. Khoo, Appl. Phys. Lett. 77, 7 (2000).

17. Y. Izdebskaya, V. Shvedov, A. Desyatnikov, W. Krolikowski, M. Belic, G. Assanto, and Y. Kivshar, Opt. Express 18, 3258 (2010).

18. J. Beeckman, K. Neyts, X. Hutsebaut, C. Cambournac, and M. Haelterman, Opt. Express 12, 1011 (2004).

19. L. Onsager, Ann. N. Y. Acad. Sci. 51, 627 (1949).

20. T. Adachi, J. Brazard, P. Chokshi, J. Bolinger, V. Ganesan, and P. Barbara, J. Phys. Chem. C 114, 20896 (2010).

21. A. Alberucci, A. Piccardi, M. Peccianti, M. Kaczmarek, and G. Assanto, Phys. Rev. A 82, 023806 (2010).

22. A. Piccardi, A. Alberucci, U. Bortolozzo, S. Residori, and G. Assanto, Appl. Phys. Lett. 96, 071104 (2010).

23. A. Piccardi, M. Trotta, M. Kwasny, A. Alberucci, R. Asquini, M. Karpierz, A. d'Alessandro, and G. Assanto, Appl. Phys. B 104, 805 (2011).

24. A. Sheridan, G. Turnbull, A. Safonov, and I. Samuel, Phys. Rev. B 62 , R11929 (2000).

25. J. Henninot, J. Blach, and M. Warenghem, J. Appl. Phys. 107, 113111 (2010).

26. A. I. Strinić, D. V. Timotijević, D. Arsenović, M. S. Petrović, and M. R. Belić, Opt. Express 13, 493 (2005).

27. J. Arrue, J. Zubia, G. Durana, and J. Mateo, IEEE J. Sel. Top. Quantum Electron. 7, 836 (2001)

28. S. Bolis, M. Celebrano, L. Ghirardini, M. Finazzi, C. Botta, J. Beeckman, P. Kockaert, and T. Virgili, Chem. Commun. 51, 9686 (2015).

29. J. B. Prieto, F. L. Arbeloa, V. M. Martínez, T. A. López, and I. L. Arbeloa, J. Phys. Chem. A 108, 5503 (2004). 


\section{FULL REFERENCES}

1. O. Svelto, S. Taccheo, and C. Svelto, "Analysis of amplified spontaneous emission: some corrections to the linford formula," Opt. Commun. 149, 277-282 (1998).

2. S. Stagira, M. Nisoli, G. Cerullo, M. Zavelani-Rossi, S. D. Silvestri, G. Lanzani, W. Graupner, and G. Leising, "The role of amplified spontaneous emission in the ultrafast relaxation dynamics of polymer films," Chem. Phys. Lett. 289, 205 (1998).

3. J. Ribierre, G. Tsiminis, S. Richardson, G. Turnbull, I. Samuel, H. Barcena, and P. Burn, "Amplified spontaneous emission and lasing properties of bisfluorene-cored dendrimers," Appl. Phys. Lett. 91, 081108 (2007).

4. G. Tsiminis, N. Montgomery, A. Kanibolotsky, A. Ruseckas, I. Perepichka, P. Skabara, G. Turnbull, and I. Samuel, "Laser characteristics of a family of benzene-cored star-shaped oligofluorenes," Semicond. Sci. Technol. 27, 094005 (2012).

5. B. Redding, M. Choma, and H. Cao, "Speckle-free laser imaging using random laser illumination," Nat. Photonics 6, 355-359 (2012).

6. B. Redding, P. Ahmadi, V. Mokan, M. Seifert, M. A. Choma, and H. Cao, "Low-spatial-coherence high-radiance broadband fiber source for speckle free imaging," Opt. Lett. 40, 4607-4610 (2015).

7. M. D. McGehee, R. Gupta, S. Veenstra, E. K. Miller, M. A. Díaz-García, and A. Heeger, "Amplified spontaneous emission from photopumped films of a conjugated polymer," Phys. Rev. B 58, 7035 (1998).

8. G. Heliotis, D. Bradley, G. Turnbull, and I. Samuel, "Light amplification and gain in polyfluorene waveguides," Appl. Phys. Lett. 81, 415-417 (2002).

9. F. Lahoz, C. J. Oton, N. Capuj, M. Ferrer-González, S. Cheylan, and D. Navarro-Urrios, "Reduction of the amplified spontaneous emission threshold in semiconducting polymer waveguides on porous silica." Opt. Express 17, 16766 (2009).

10. E. Calzado, M. Ramírez, P. Boj, and M. Díaz-García, "Thickness dependence of amplified spontaneous emission in low-absorbing organic waveguides," Appl. Opt. 51, 3287 (2012).

11. S. Bolis, M. Pasini, and T. Virgili, "A core copolymer approach to improve the gain properties of a red-emitting molecule," Chem. Commun. 49, 11761-11761 (2013).

12. K. Vishnubhatla, J. Clark, G. Lanzani, R. Ramponi, R. Osellame, and T. Virgili, "Ultrafast optofluidic gain switch based on conjugated polymer in femtosecond laser fabricated microchannels," Appl. Phys. Lett. 94, 041123 (2009).

13. R. M. Gerosa, A. Sudirman, L. d. S. Menezes, W. Margulis, and C. J. de Matos, "All-fiber high repetition rate microfluidic dye laser," Optica 2, 186-193 (2015).

14. L. Blinov, G. Cipparrone, V. Lazarev, P. Pagliusi, and T. Rugiero, "Liquid crystal as laser medium with tunable gain spectra," Opt. Express 16, 6625-6630 (2008).

15. R. Fan, Y. Jiang, Y. Xia, and D. Chen, "Widely tunable amplified spontaneous emission of pyrromethene-650-doped nematic liquid crystal," J. Russ. Laser Res. 32, 139-143 (2011).

16. M. Peccianti, A. D. Rossi, G. Assanto, A. D. Luca, C. Umeton, and I. Khoo, "Electrically assisted self-confinement and waveguiding in planar nematic liquid crystal cells," Appl. Phys. Lett. 77, 7-9 (2000).

17. Y. Izdebskaya, V. Shvedov, A. Desyatnikov, W. Krolikowski, M. Belic, G. Assanto, and Y. Kivshar, "Counterpropagating nematicons in biasfree liquid crystals," Opt. Express 18, 3258-3263 (2010).

18. J. Beeckman, K. Neyts, X. Hutsebaut, C. Cambournac, and M. Haelterman, "Simulations and experiments on self-focusing conditions in nematic liquid-crystal planar cells," Opt. Express 12, 1011 (2004).

19. L. Onsager, "The effects of shape on the interaction of colloidal particles," Ann. N. Y. Acad. Sci. 51, 627 (1949).

20. T. Adachi, J. Brazard, P. Chokshi, J. Bolinger, V. Ganesan, and P. Barbara, "Highly ordered single conjugated polymer chain rod morphologies," J. Phys. Chem. C 114, 20896 (2010).

21. A. Alberucci, A. Piccardi, M. Peccianti, M. Kaczmarek, and G. Assanto, "Propagation of spatial optical solitons in a dielectric with adjustable nonlinearity," Phys. Rev. A 82, 023806 (2010).

22. A. Piccardi, A. Alberucci, U. Bortolozzo, S. Residori, and G. Assanto,
"Soliton gating and switching in liquid crystal light valve," Appl. Phys. Lett. 96, 071104 (2010).

23. A. Piccardi, M. Trotta, M. Kwasny, A. Alberucci, R. Asquini, M. Karpierz, A. d'Alessandro, and G. Assanto, "Trends and trade-offs in nematicon propagation," Appl. Phys. B 104, 805-811 (2011).

24. A. Sheridan, G. Turnbull, A. Safonov, and I. Samuel, "Tuneability of amplified spontaneous emission through control of the waveguidemode structure in conjugated polymer films," Phys. Rev. B 62, R11929 (2000).

25. J. Henninot, J. Blach, and M. Warenghem, "Enhancement of dye fluorescence recovery in nematic liquid crystals using a spatial optical soliton," J. Appl. Phys. 107, 113111 (2010).

26. A. I. Strinić, D. V. Timotijević, D. Arsenović, M. S. Petrović, and M. R. Belić, "Spatiotemporal optical instabilities in nematic solitons," Opt. Express 13, 493-504 (2005).

27. J. Arrue, J. Zubia, G. Durana, and J. Mateo, "Parameters affecting bending losses in graded-index polymer optical fibers," IEEE J. Sel. Top. Quantum Electron. 7, 836-844 (2001).

28. S. Bolis, M. Celebrano, L. Ghirardini, M. Finazzi, C. Botta, J. Beeckman, P. Kockaert, and T. Virgili, "Optical gain from polyfluorene keto defects in a liquid crystal mix," Chem. Commun. 51, 9686 (2015).

29. J. B. Prieto, F. L. Arbeloa, V. M. Martínez, T. A. López, and I. L. Arbeloa, "Photophysical properties of the pyrromethene 597 dye: solvent effect," J. Phys. Chem. A 108, 5503-5508 (2004). 